\title{
Near-Capacity Non-Coherent Network-Coding Aided Scheme for Cooperative Multi-user Communications
}

\author{
Hung Viet Nguyen ${ }^{1}$, Chao $\mathrm{Xu}^{1}$, Soon Xin $\mathrm{Ng}^{1}$, João Luiz Rebelatto ${ }^{2}$, Yonghui $\mathrm{Li}^{3}$ and Lajos Hanzo ${ }^{1}$ \\ ${ }^{1}$ School of Electronics and Computer Science, University of Southampton, SO17 1BJ, United Kingdom. \\ 2 Dept. of Electrical Engineering, Federal University of Santa Catarina, Florianópolis, SC, 88040-900, Brazil. \\ ${ }^{3}$ School of Electrical \& Information Engineering, University of Sydney, Sydney, NSW, 2006, Australia. \\ Emails: ${ }^{1}\left\{\right.$ hvn08r,cx1g08,sxn,lh\}@ecs.soton.ac.uk; ${ }^{2}$ jlrebelatto@eel.ufsc.br; ${ }^{3}$ lyh@ee.usyd.edu.au
}

\begin{abstract}
In this contribution, Near-capacity Non-coherent Cooperative Network-coding aided Multi-user (NNCNM) systems are designed with the aid of Extrinsic Information Transfer (EXIT) charts for the sake of approaching the Differential Discrete-input Continuous-output Memoryless Channel (D-DCMC)-based capacity. The proposed subframe-based network coding solution allows the system to significantly mitigate the effects of large-scale fading on each frame. Hence, NNCNM systems operating in large-scale fading environments are capable of approaching the D-DCMC capacity of the less hostile single link channel incurring the small-scale fading, but no shadow fading.
\end{abstract}

\section{INTRODUCTION}

Network coding is a recently introduced paradigm conceived for efficiently disseminating data in multicast wireless networks, where the data flows arriving from multiple sources are combined to achieve compression and hence to increase the achievable throughput, as well as to reduce the delay imposed and to enhance the error-resilience [1], [2].

The Dynamic Network Code (DNC) concept proposed in [3], [4] was extended in [5], [6] in order to introduce Generalised Dynamic Network Codes (GDNC). In GDNC aided systems, each user is allowed to broadcast several (as opposed to a single in [3], [4]) information frames (IF) of its own during the broadcast phase (BP) via orthogonal channels, as well as to transmit several nonbinary linear combinations, which are also considered as parity frames (PFs), during the cooperative phase (CP) via orthogonal channels. The FER performance of the GDNC scheme was determined in [5], [6] by calculating the rank of the matrix characterising GDNCs. This method, which we refer to as the Purely Rank-Based Method (PRBM), always provides an optimistic estimate of the attainable FER performance of GDNCs.

Tüchler and Hagenauer proposed the employment of Irregular Convolutional Codes (IrCCs) [7] for serially concatenated schemes, which are constituted by a family of convolutional codes having different rates, in order to design a near-capacity system. They were specifically designed with the aid of Extrinsic Information Transfer (EXIT) charts conceived for analysing the convergence properties of iterative decoding aided concatenated coding schemes [8].

As the number of sources and relays increases, it becomes unrealistic to obtain accurate Channel State Information (CSI) for the increasing number of mobile-to-mobile channels, which may erode the performance of the near capacity coherent modulation schemes designed with the aid of EXIT charts relying on the assumption of perfect CSI estimation. Hence Differential M-ary Phase-Shift Keying (DMPSK) can be chosen for the sake of eliminating the excessive complexity of channel estimation in distributed networks.

Furthermore, Multiple-Symbol Differential Detection (MSDD) [9] may be employed in order to mitigate the performance loss of the non-coherent receivers. Since the differential encoder is 'recursive' -

The financial support of the Vietnamese International Education Development (VIED) fund and the EU under the auspices of the OPTIMIX project as well as of the RC-UK under the India-UK Advanced Technology Centre is gratefully acknowledged. i.e. has an infinite impulse response - similar to Recursive Systematic Convolutional (RSC) codes, the $(1,1)$ point of the EXIT chart can be approached by MSDD having a detection window length as long as the decoding frame length. However, the MSDD window length is severely limited, because its extension imposes an exponentially increasing detection complexity. As a remedy, Multiple-Symbol Differential Sphere Detection (MSDSD) [10] was proposed for reducing the complexity, but the employment of a frame-sized detection window length still remains impractical. Furthermore, having a slightly degraded performance is unavoidable, since MSDSD constitutes the Max-Log-MAP - rather than MAP - algorithm of MSDD. Therefore, a recursive Unity-Rate Code (URC) [11] having an infinite impulse response may be employed as an intermediate code. In other words, a near-capacity channel coding scheme - namely IrCC-URC-DMPSK associated with MSDD - can be involved for the sake of approaching the achievable non-coherent-detection channel capacity, while the high-complexity, yet imperfect channel estimation is eliminated.

Hence, the novel contribution of this paper is that a near-capacity channel coding scheme is designed for the sake of supporting cooperative non-coherent communications. More specifically, the near capacity coded differential modulation scheme associated with MSDD is employed in the network coding based system, in order to achieve good performance without channel estimation. We consider the effects of both the shadow fading and of the small-scale Rayleigh fading in our channel model. The performance of the proposed system evaluated by simulations is compared to that estimated by PRBM. The design guidelines presented in this contribution can also be extended to a diverse range of network-coding aided multiuser systems employing arbitrary channel coding schemes.

\section{Single Link Channel Outage Probability}

We consider a single transmission link associated with the transmitted and received signals of $x$ and $y$, respectively. The received signal can be represented as

$$
y=h x+n
$$

where $h=h_{s} h_{f}$ is the complex-valued fading coefficient that comprises of two components, a large-scale shadow fading $h_{s}$, which is constant for all symbols within a transmission frame and a smallscale Rayleigh fading coefficient $h_{f}$, which varies on a symbol by symbol basis, while $n$ is the Additive White Gaussian Noise (AWGN) process having a variance of $N_{0} / 2$ per dimension.

The outage probability for the CCMC channel is given by [12]

$$
P_{e}^{C C M C}(R)=\operatorname{Pr}\left\{\left|h_{s}\right|^{2} \mathrm{E}\left[\left|h_{f}\right|^{2}\right]<\frac{2^{R}-1}{S N R}\right\},
$$

where $S N R$ is the signal to noise power ratio, while assuming that the transmitter encodes data at a rate of $R$ bits/s/Hz.

We define the receiver's $S N R$ as $S N R_{r}=E\left[|h|^{2} S N R\right]$, and consider a Differential Discrete-input Continuous-output Memoryless Channel (D-DCMC) having a data rate of $R=\eta R_{c}$, where $\eta$ is the number of modulated bits and $R_{c}$ is the equivalent channel coding 
rate. At a given data rate $R$, we can identify the corresponding $\left.S N R_{r}\right|_{R}$ point on the D-DCMC capacity curve, which will be determined by using EXIT charts in Section V-A. Then, similar to (2), the outage probability of the D-DCMC model may be formulated as:

$$
P_{e}^{D-D C M C}(R, \eta)=\operatorname{Pr}\left\{\left|h_{s}\right|^{2} \mathrm{E}\left[\left|h_{f}\right|^{2}\right]<\frac{\left.S N R_{r}\right|_{R}}{S N R}\right\} .
$$

\section{MSDD-AIDED-DMPSK}

For DMPSK schemes, differential encoding is carried out according to:

$$
s_{n}= \begin{cases}s_{1} & n=1 \\ x_{n-1} s_{n-1} & n>1\end{cases}
$$

where $x_{n}$ carries the source information. For a single transmission link, the signal received over a Rayleigh fading channel may be expressed as:

$$
y_{n}=s_{n} h_{n}+n_{n},
$$

where the AWGN $n_{n}$ has a zero mean and a variance of $N_{0}$, while $h_{n}$ denotes the fading coefficient having a temporal correlation of $\varepsilon\left\{h_{n} h_{n+k}^{*}\right\}=J_{0}\left(2 \pi k f_{d}\right)$ according to Clarke's fading model [9], where $J_{0}$ denotes the zero-order Bessel function of the first kind and $f_{d}$ is the normalized Doppler frequency.

In order to observe the received signal across an MSDD decision window of $N_{w}$ consecutive symbols, (5) may be further developed as:

$$
\mathbf{y}=\mathbf{s h}+\mathbf{n},
$$

where the $\left(N_{w} \times 1\right)$-element matrix $\mathbf{y}=\left[y_{n-N_{w}+1}, \cdots, y_{n}\right]^{T}$ models the received symbols within the MSDD window, while the equivalent fading channel matrix $\mathbf{h}=\left[h_{n-N_{w}+1}, \cdots, h_{n}\right]^{T}$ and the equivalent AWGN matrix $\mathbf{n}=\left[n_{n-N_{w}+1}, \cdots, n_{n}\right]^{T}$ are both of size $\left(N_{w} \times 1\right)$. The $\left(N_{w} \times N_{w}\right)$-element equivalent transmission matrix $\mathbf{s}$ of (6) is modelled as $\mathbf{s}=\operatorname{diag}\left\{\left[s_{n-N_{w}+1}, \cdots, s_{n}\right]\right\}^{T}$.

The MSDD aims for minimizing the a posteriori probability of [9]:

$$
\operatorname{Pr}(\mathbf{y} \mid \mathbf{s})=\frac{\exp \left[-\operatorname{tr}\left\{\mathbf{y}^{H}\left(\mathbf{R}_{\mathbf{y y}}\right)^{-1} \mathbf{y}\right\}\right]}{\pi^{N_{w}} \operatorname{det}\left(\mathbf{R}_{\mathbf{y y}}\right)},
$$

where the correlation matrix $\mathbf{R}_{\mathbf{y y}}$, whose determinant is a real-valued constant, is given by:

$$
\mathbf{R}_{\mathbf{y y}}=\mathbf{s R}_{\mathbf{h h}} \mathbf{s}^{H}+\mathbf{R}_{\mathbf{n n}}=\mathbf{s} \mathbf{C s}^{H},
$$

where the correlation of the fading channel is given by $\mathbf{R}_{\mathbf{h h}}=$ Toeplitz $\left\{\rho_{0}, \cdots, \rho_{N_{w}-1}\right\}^{1}$. with $\rho_{k}=N J_{0}\left(2 \pi k f_{d}\right)$, while the correlation matrix of the AWGN is given by $\mathbf{R}_{\mathbf{n n}}=N_{0} \cdot \mathbf{I}_{\mathbf{N}_{\mathbf{w}}}$. The channel correlation matrix in (8) is defined as $\mathbf{C}=\mathbf{R}_{\mathbf{h h}}+\mathbf{R}_{\mathbf{n n}}$. Therefore, the trace operation in (7) may be further formulated as:

$$
\operatorname{tr}\left\{\mathbf{y}^{H}\left(\mathbf{R}_{\mathbf{y y}}\right)^{-1} \mathbf{y}\right\}=\operatorname{tr}\left\{\mathbf{y}^{H} \mathbf{s C}^{-1} \mathbf{s}^{H} \mathbf{y}\right\}=\left\|\mathbf{L}^{H} \mathbf{s}^{H} \mathbf{y}\right\|^{2},
$$

where the lower triangle matrix $\mathbf{L}$ is generated by the decomposition of $\mathbf{C}^{-1}=\mathbf{L L}^{H}$. Based on the a posteriori probability of (7), the Log-MAP algorithm conceived for MSDD-aided-DMPSK may be

$L_{e}\left(b_{k} \mid \mathbf{y}\right)=\ln \left(\frac{\sum_{\mathbf{s} \in \mathbf{s}_{1}^{k}} \exp \left[\left\|\mathbf{L}^{H} \mathbf{s}^{H} \mathbf{y}\right\|^{2}+\sum_{j=1, j \neq k}^{N_{w}\left(\log _{2} M\right)} b_{j} L_{a}\left(b_{j}\right)\right]}{\sum_{\mathbf{s} \in \mathbf{s}_{0}^{k}} \exp \left[\left\|\mathbf{L}^{H} \mathbf{s}^{H} \mathbf{y}\right\|^{2}+\sum_{i=1, i \neq k}^{N_{w}\left(\log _{2} M\right)} b_{i} L_{a}\left(b_{i}\right)\right]}\right)$

where $L_{e}\left(b_{k} \mid \mathbf{y}\right)$ denotes the extrinsic LLR provided for the bit $b_{k}$, while $\mathbf{s}_{0}^{k}$ and $\mathbf{s}_{1}^{k}$ refer to the constellation set corresponding to the equivalent transmission matrix $\mathbf{s}$ when $b_{k}$ is set to 0 and 1 , respectively.

${ }^{1}$ This notation simply indicates that $R_{h h}$ is a Toeplitz-structured matrix constituted by the elements $\left\{\rho_{0}, \cdots, \rho_{N_{w}-1}\right\}$

\section{System MOdel}

\section{A. System description}

Let us initially describe a simple system having $M=2$ users communicating with a BS [3]. A transmission session consists of $\left(k_{1} M+k_{2} M\right)=4$ phases that include broadcast phases $B_{1}$ and $B_{2}$ and cooperative phases $C_{1}$ and $C_{2}$. In the transmission session, each user transmits $k_{1}=1 \mathrm{IF}$ during the corresponding broadcast phase and $k_{2}=1 \mathrm{PF}$ during the corresponding cooperative phase according to the transfer matrix $\boldsymbol{G}_{2 \times 4}$ [3], [4]:

$$
\boldsymbol{G}_{2 \times 4}=\left[\begin{array}{ccc|cc}
1 & 0 & 1 & 1 \\
0 & 1 & \mid & 1 & 2
\end{array}\right],
$$

where the PF transmitted by User 1 (or User 2) during the cooperative phase $C_{1}$ (or $C_{2}$ ) is given by $\mathrm{PF}=\boldsymbol{G}_{2 \times 4}(1,3) I_{1}(1)+\boldsymbol{G}_{2 \times 4}(2,3) I_{2}(2)$ (or $\mathrm{PF}=\boldsymbol{G}_{2 \times 4}(1,4) I_{1}(1)+\boldsymbol{G}_{2 \times 4}(2,4) I_{2}(2)$ ). The variable $I_{i}(i), i=$ $[1,2]$, represents the IF transmitted by User $i$ during the broadcast phase $B_{i}$. For simplicity, we refer to a single transmission phase ( broadcast phase or cooperative phase) as a time slot (TS), in which a user transmits a single frame (IF or PF).

Furthermore, let us define $\boldsymbol{G}_{2 \times 4}^{\prime}$ as the corresponding modified transfer matrix, where the terminology modified implies that the entries of $\boldsymbol{G}_{2 \times 4}^{\prime}$ are modified with respect to those of the original transfer matrix $\boldsymbol{G}_{2 \times 4}$ of (11) according to the success/failure of each transmission within the actual transmission session. If all the frames transmitted within the session are successfully decoded, the transmission session can be represented by the modified transfer matrix $\boldsymbol{G}_{2 \times 4}^{\prime}=\boldsymbol{G}_{2 \times 4}$, where $\boldsymbol{G}_{2 \times 4}^{\prime}(i, i)=\boldsymbol{G}_{2 \times 4}(i, i), i=[1,2]$ represents the successful decoding of the IF $I_{i}(i)$ at the BS. Note that having $\boldsymbol{G}_{2 \times 4}^{\prime}(1,3)=\boldsymbol{G}_{2 \times 4}(1,3)\left(\right.$ or $\boldsymbol{G}_{2 \times 4}^{\prime}(2,4)=\boldsymbol{G}_{2 \times 4}(2,4)$ ) means that the PF transmitted by User 1 (or User 2) was successfully decoded at the BS. Similarly, having $\boldsymbol{G}_{2 \times 4}^{\prime}(2,3)=\boldsymbol{G}_{2 \times 4}(2,3)$ (or $\boldsymbol{G}_{2 \times 4}^{\prime}(1,4)=\boldsymbol{G}_{2 \times 4}(1,4)$ ) indicates that the IF $I_{2}(2)$ (or $I_{1}(1)$ ) was successfully decoded by User 1 (or User 2), and that the PF transmitted by User 1 (or User 2) was successfully decoded at the BS.

Let us consider the following example of the actual transmission session, where ${ }^{\prime} \rightarrow{ }^{\prime}$ represents the transmission direction, while ${ }^{\prime}=$ $1^{\prime}\left(\right.$ or $\left.^{\prime}=0^{\prime}\right)$ above the arrows means that the frame was successfully (or unsuccessfully) recovered at the destination:

$$
\begin{array}{ll}
B_{1} \quad \boldsymbol{G}_{2 \times 4}^{\prime}(1,3)=\boldsymbol{G}_{2 \times 4}(1,3), \\
& {[\text { User } 1 \stackrel{=0}{\longrightarrow} B S]: \boldsymbol{G}^{\prime}(1,1)=0,} \\
& {[\text { User } 1 \stackrel{=1}{\longrightarrow} \text { User } 2]: \boldsymbol{G}_{2 \times 4}^{\prime}(1,4)=\boldsymbol{G}_{2 \times 4}(1,4),} \\
B_{2} \quad & \boldsymbol{G}_{2 \times 4}^{\prime}(2,4)=\boldsymbol{G}_{2 \times 4}(2,4), \\
& {[\text { User } 2 \stackrel{=0}{\longrightarrow} B S]: \boldsymbol{G}^{\prime}(2,2)=0,} \\
& {[\text { User } 2 \stackrel{=1}{\longrightarrow} \text { User } 1]: \boldsymbol{G}_{2 \times 4}^{\prime}(2,3)=\boldsymbol{G}_{2 \times 4}(2,3),} \\
C_{1} & {[\text { User } 1 \stackrel{=0}{\longrightarrow} B S]: \boldsymbol{G}_{2 \times 4}^{\prime}(i, 3)=0, i=1,2,} \\
C_{2} & {[\text { User } 2 \stackrel{=1}{\longrightarrow} B S]: \boldsymbol{G}_{2 \times 4}^{\prime}(i, 4) \text { unchanged, } i=1,2 .}
\end{array}
$$

This example results in

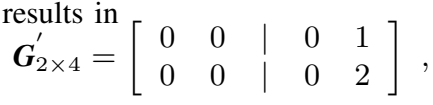

where the diagonal elements "1" at the left of (11) become "0" , owing to the unsuccessful [User $1 \stackrel{=0}{\longrightarrow} B S$ ] and [User $2 \stackrel{=0}{\longrightarrow} B S$ ] transmissions during the broadcast phases $B_{1}$ and $B_{2}$, respectively. The " 0 " elements in the third column of (13) indicate the unsuccessful [User $1 \stackrel{=0}{\longrightarrow} B S$ ] transmission during the cooperative phase $C_{1}$.

Let us now generalise this model. The transfer matrix $\boldsymbol{G}_{k_{1} M \times k_{1} M+k_{2} M}$ (or $\boldsymbol{G}$ for shorthand) seen in Fig. 1, which comprises the identity matrix $\boldsymbol{I}_{k_{1} M \times k_{1} M}$ (or $\boldsymbol{I}$ for shorthand) and the parity matrix $\boldsymbol{P}_{k_{1} M \times k_{2} M}$ (or $\boldsymbol{P}$ for shorthand) represents a transmission session of the system, where all the frames transmitted during 
that session are successfully decoded. The binary flag $I_{m}^{C o}(t)$ seen in Fig. 1 represents the success or failure of the IF decoding at the BS, namely the IF $I_{m}(t), t=\left[m, M+m, \ldots,\left(k_{1}-1\right) M+m\right]$, transmitted by User $m, m \in\{1, \ldots, M\}$. Accordingly, we always have $I_{m}^{C o}(t)=1$. The corresponding entry $I_{m}^{C o^{\prime}}(t)$ is set during the specific broadcast phase $t$ selected from the whole set of $k_{1} M$ broadcast phases according to [5], [6]:

$I_{m}^{C o}(t)=\left\{\begin{array}{rll}I_{m}^{C o}(t) & : & \text { If } I_{m}(t) \text { is successfully recovered } \\ 0 & : & \text { Otherwise }\end{array}\right.$

The $k_{2}$ PFs transmitted by each of the $M$ users contain nonbinary linear combinations of its own IFs with the successfully decoded IFs from the set of $k_{1}(M-1)$ IFs transmitted by the $(M-1)$ other users. The variable $P_{m, s}(t)$ in Fig. 1 corresponds to the parity coefficient of the IF $I_{r}(t)$ contained in the $s^{t h}$ PF transmitted by User $m$ during the cooperative phase $[M(s-1)+m], s \in\left\{1, \ldots, k_{2}\right\}$, where we have the index $r$ determined by

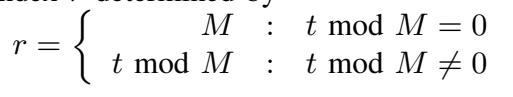

Let us denote the corresponding entry of $P_{m, s}(t)$ in the modified matrix $\boldsymbol{G}^{\prime}$ as $P_{m, s}^{\prime}(t)$, which is determined by

$$
P_{m, s}^{\prime}(t)=\left\{P_{m, s}(t) \quad: \quad r=m .\right.
$$

Then, for the case that we have $r \neq m$, the entry $P_{m, s}^{\prime}(t)$ is specified by [5], [6]

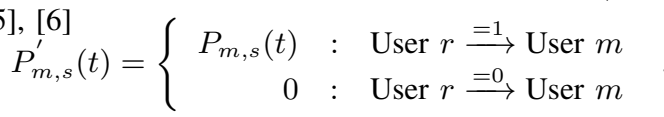

The column $[M(s-1)+m]$ of the parity matrix $\boldsymbol{P}$ shown in Fig. 1 contains the set of parity coefficients valid for the $s^{\text {th }}$ PF transmitted by User $m$ during the cooperative phase $[M(s-1)+m]$. Hence, the entire column $P_{m, s}^{\prime}(t), \forall t=\left[1,2, \ldots, k_{1} M\right]$ will be set to zeros, if the BS could not successfully receive the $s^{\text {th }} \mathrm{PF}$ :

$$
P_{m, s}^{\prime}(t)=0, \forall t=\left[1,2, \ldots, k_{1} M\right]: \text { User } m \stackrel{s^{t h} \mathrm{PF}=0}{\longrightarrow} \mathrm{BS} .
$$

\section{B. Detection model}

As the system proceeds through an actual transmission session, the corresponding modified transfer matrix $\boldsymbol{G}^{\prime}$ consisting of its identity matrix $\boldsymbol{I}^{\prime}$ and its parity matrix $\boldsymbol{P}^{\prime}$ is formed, where $\boldsymbol{I}^{\prime}$ is generated from (14), while $\boldsymbol{P}^{\prime}$ is determined in turn by (16), (17) and (18). The frames successfully received at the BS can be represented as

$$
\text { (a) } \boldsymbol{X} \boldsymbol{I}^{\prime}=\boldsymbol{Y}_{I^{\prime}}, \text { (b) } \boldsymbol{X} \boldsymbol{P}^{\prime}=\boldsymbol{Y}_{P^{\prime}}
$$

where $\boldsymbol{X}=\left\{I_{1}(1), I_{2}(2), \ldots, I_{M}\left(k_{1} M\right)\right\}$ is a matrix representing the IFs transmitted by the $M$ users during the transmission session of the system, while the matrices of $\boldsymbol{Y}_{I^{\prime}}$ and $\boldsymbol{Y}_{P^{\prime}}$ represent the frames successfully received at the BS during the broadcast phases and cooperative phases, respectively. In line with [5], [6], we assume that the BS is aware of how each PF was constructed, hence $\boldsymbol{G}^{\prime}$ is known at the BS. Since the matrix $\boldsymbol{I}^{\prime}$ may be different from $\boldsymbol{I}$, the BS can certainly recover a set $\boldsymbol{X}_{I^{\prime}}$ of IFs, which is a subset of $\boldsymbol{X}$, from $\boldsymbol{Y}_{I^{\prime}}$ according to: $\boldsymbol{X}_{I^{\prime}}=\boldsymbol{Y}_{I^{\prime}}$.

Substituting $\boldsymbol{X}_{I^{\prime}}$ given by (20) into (19b) we, have

$$
\left(\boldsymbol{X}-\boldsymbol{X}_{I^{\prime}}\right) \boldsymbol{P}^{\prime}=\boldsymbol{Y}_{P^{\prime}}-\boldsymbol{X}_{I^{\prime}} \boldsymbol{P}^{\prime} .
$$

Then, a set $\tilde{\boldsymbol{X}}_{P^{\prime}}$ of IFs is retrieved from (21) by using the Gaussian elimination algorithm [13]. Ultimately, the entire set of IFs recovered at the BS is $\tilde{\boldsymbol{X}}_{P^{\prime}} \cup \boldsymbol{X}_{I^{\prime}}$ out of the $\boldsymbol{X}$ of IFs.

Having presented the detection model above, let us now characterise the system's optimistic performance estimated by the PRBM employed in [5], [6] by recalling the example detailed in (11) and (12). According to the prediction of the PRBM, the BS can recover $\operatorname{Rank}\left(\boldsymbol{G}_{2 \times 4}^{\prime}\right)=1 \mathrm{IF}$, where $\boldsymbol{G}_{2 \times 4}^{\prime}$ is given in (13). However, in fact the BS cannot recover any IF, because we cannot unambiguously determine two IFs, i.e. both $I_{1}(1)$ and $I_{2}(2)$, from a single equation, which is inferred from (20) and (21) as $1 \times I_{1}(1)+2 \times I_{2}(2)$.

\section{Design And AnAlysis}

\section{A. Near-Capacity Code Design}

According to (1) and (2), the average $S N R_{r}$ per frame can be expressed as

$$
S N R_{r}=\frac{\mathrm{E}\left[\left|h_{s}\right|^{2}\right] \mathrm{E}\left[\left|h_{f}\right|^{2}\right] \mathrm{E}\left[|x|^{2}\right]}{N_{0}}=\frac{\left|h_{s}\right|^{2}}{N_{0}},
$$

where we have $\mathrm{E}\left[|x|^{2}\right]=1$ and $\mathrm{E}\left[\left|h_{s}\right|^{2}\right]=\left|h_{s}\right|^{2}$ for correlated Rayleigh fading channels. Given a specific $S N R_{r}$, we can generate the EXIT chart [8] of the system. As stated in Section I, a near-

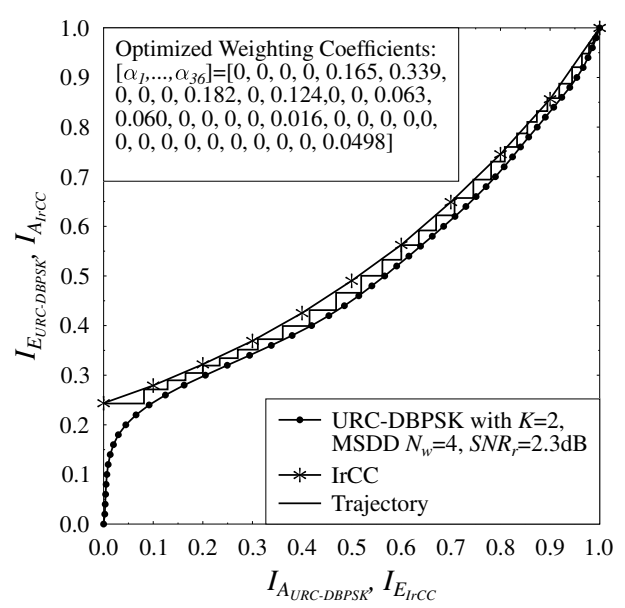

Fig. 2. The EXIT curves of the inner decoder URC-MSDD-aided-DBPSK and the outer decoder IrCC along with the Monte-Carlo simulation based decoding trajectory when $f_{d}=0.03$.

capacity IrCC-URC-MSDD-aided-DMPSK channel coding scheme is chosen for the sake of approaching the achievable channel capacity. For the sake of simplicity, we present our generic design procedure for the specific example of IrCC-URC-Differential Binary Phase Shift Keying (IrCC-URC-DBPSK) associated with MSDD $N_{w}=4$ using our generically applicable EXIT-chart aided method, which is briefly summarised as follows:

Step1: Create the EXIT curve of the inner decoder component constituted by our URC-MSDD-aided-DBPSK scheme for different $S N R_{r}$ values;

Step2: We opt for the data rate $R=\eta \times R_{c}=2 \times 0.5$, in order to facilitate a comparison between our results and the previous results presented in [5], [6]. We then fix the IrCC code rate $R_{c}=0.5$ and employ the EXIT curve matching algorithm of [14] for generating the optimised weighting coefficients $\alpha_{i}, i=1, \ldots, 36$, of the 36 differentrate component IrCC codes. More specifically, we opt for the set of codes facilitating decoding convergence to a vanishingly low $B E R$ at the lowest possible $S N R_{r}$, while ensuring that the Monte-Carlo simulation based decoding trajectory reaches the point of $(1,1)$ at the top-right corner of the corresponding EXIT chart. This implies that a near-capacity performance can be achieved, as detailed in [15].

Having implemented the design steps mentioned above, we obtain the EXIT curves and the corresponding IrCC component-code weighting coefficients $\alpha_{i}, i=1, \ldots, 36$, as shown in Fig. 2. Again as detailed in [15], these weighting coefficients $\alpha_{i}$ determine the particular fraction of the input stream to be encoded by the $i^{\text {th }}$ IrCC component code having a code rate of $\alpha_{i}$. The EXIT-chart results show that provided a sufficiently high number of iterations, say $J$ is carried out between the IrCC decoder and the composite URCMSDD-aided-DBPSK decoder, the Monte-Carlo simulation based decoding trajectory would reach the $(1,1)$ point in Fig. 2, which guarantees a vanishingly low $B E R$. 


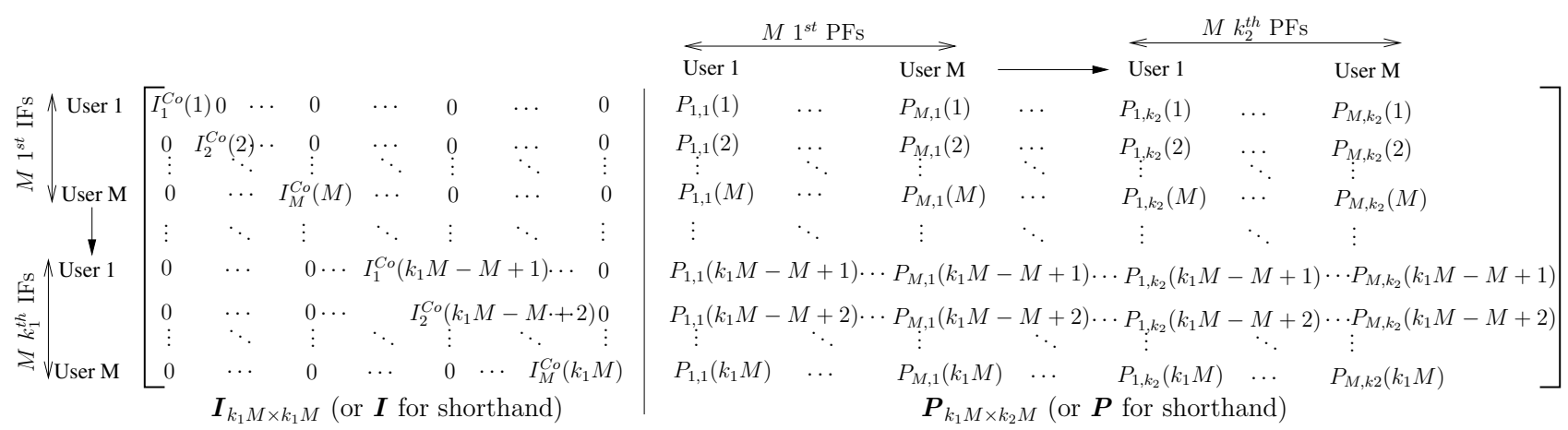

Fig. 1. The transfer matrix $\boldsymbol{G}_{k_{1} M \times k_{1} M+k_{2} M}$ [5], [6] illustrating a transmission session of the system having $M$ users transmitting in $\left(k_{1} M+k_{2} M\right)$ phases.

Furthermore, the area property of EXIT-charts [16] states that the area under the EXIT curve of an inner decoder component is approximately equal to the attainable channel capacity, provided that the channel's input symbols are equiprobable. Hence we exploited the area property of EXIT-charts [16] to determine the achievable capacities of the URC-MSDD-aided-DBPSK and IrCC-URC-MSDDaided-DBPSK systems, which are quantified in Fig. 3. It is seen in Fig. 3 that the capacity curve of the URC-MSDD-aided-DBPSK scheme approaches that of the MSDD-aided-DBPSK arrangement, when $K>1$ inner iterations are employed for the composite URCMSDD-aided-DBPSK decoder. It is also demonstrated in Fig. 3 that the attainable capacity improvement becomes negligible for $K>2$. Therefore, we fix the number of inner iterations to $K=2$ throughout this paper. The numerical results of Fig. 3 also show that for a sufficiently high number of say $J \geq 30$ outer iterations, the distance between the capacity curve of the IrCC-URC-MSDD-aided-DBPSK scheme and that of the DCMC-MSDD-aided-DBPSK arrangement is less than $0.5 \mathrm{~dB}$.

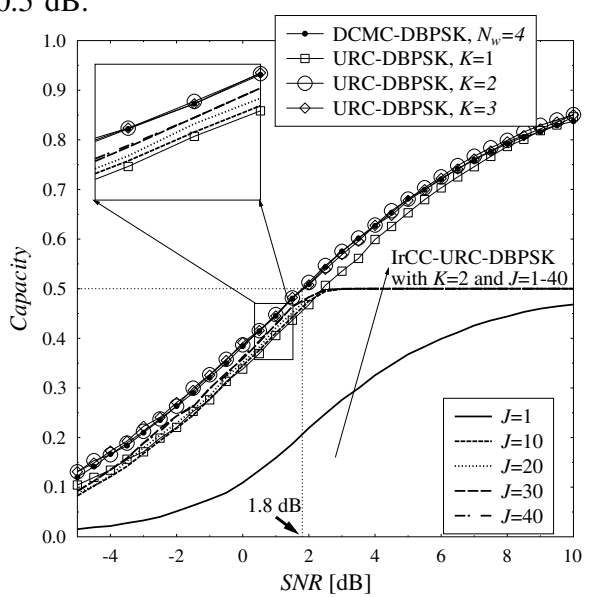

Fig. 3. Channel capacity comparison for the MSDD-aided-DBPSK, URCMSDD-aided-DBPSK and IrCC-URC-MSDD-aided-DBPSK based systems when $f_{d}=0.03$.

Our simulation results seen in Fig. 4 verify the accuracy of our EXIT chart analysis. When employing $J=30$ outer iterations between the IrCC and URC components, our IrCC-URC-MSDDaided-DBPSK channel coding scheme has a vanishingly low BER for $S N R \mathrm{~s}$ in excess of $5.1 \mathrm{~dB}$, provided that the transmission frame length is sufficiently high. Therefore, we opted for a frame length of $10^{6}$ symbols for our system. At this stage we also define the relaying-aided reduced-distance-related pathloss-reduction. Naturally, this pathloss-reduction becomes unity for each direct source-todestination link [17].

Moreover, in the practical systems, each channel-encoded frame tends to be transmitted in an $N$ number of sub-frames, where the average $S N R_{r}$ of (22) may be formulated as:

$$
S N R_{r}=\frac{\mathrm{E}\left[\left|h_{s}\right|^{2}\right]}{N_{0}}=\frac{\sum_{i=1}^{N}\left|h_{s, i}\right|^{2} / N}{N_{0}},
$$

with $h_{s, i}$ representing the large-scale shadow fading corresponding to the $i^{t h}$ sub-frame of the channel-encoded frame. As seen in Fig. 4, while the number $N$ of the sub-frames is increasing from $N=1$ to $N=10^{4}$, the performance of the IrCC-URC-MSDD-aided-DBPSK scheme recorded for transmission over a channel, where both the large-scale shadow fading and the small-scale Rayleigh fading are taken into consideration, approaches that of the IrCC-URC-MSDDaided-DBPSK scheme communicating over the small-scale Rayleigh fading channel.

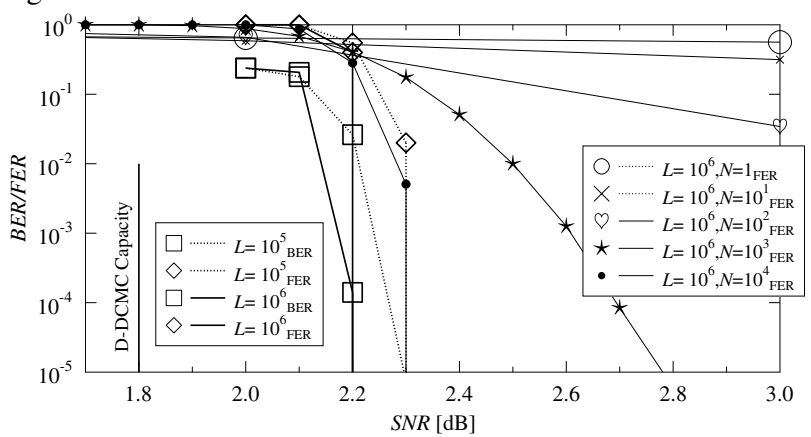

Fig. 4. Performance of the proposed IrCC-URC-MSDD-aided-DBPSK scheme in the small-scale Rayleigh fading channel with $f_{d}=0.03$ as well as in the large-scale shadow fading channel, the number of sub-frames from $N=1$ to $N=10^{4}$.

\section{B. Network Coding Design}

In line with [5], [6], we assume that all the links in the Noncoherent Near-capacity Cooperative Network-coding based Multiuser (NNCNM) system are supported by channels having the same data rate $R$. For notational convenience, we characterise our proposed NNCNM system by using the set of parameters $\left(R, M, k_{1}, k_{2}, \boldsymbol{G}, R_{\text {info }}, D_{N N C N M}\right)$, where the system's overall data rate $R_{\text {info }}$ is expressed as [5], [6] $k_{1}$

$$
R_{\text {info }}=\frac{k_{1}}{k_{1}+k_{2}},
$$

while the diversity order $D$ of the system is bounded [3], [5], [6]:

$$
M+k_{2} \leq D_{N N C N M} \leq M k_{2}+1 .
$$

By observing the $R_{\text {info }}$ expression of (24) and the $D_{N N C N M}$ formula of (25), it is plausible that we may conceive different systems having the same rate $R_{N N C N M}$, but different diversity order $D_{N N C N M}$ by independently adjusting $k_{1}, k_{2}$ and $M$. In other words, using (24) and (25), we are able to design a network-coding based system having the highest possible diversity order at a given overall system data rate of $R_{N N C N M}$. A higher diversity order implies that the system is capable of achieving an improved FER performance.

In order to demonstrate the generic design principles mentioned above, let us now consider a specific $\boldsymbol{G}_{2 \times 4}$-based system and a $\boldsymbol{G}_{4 \times 8^{-}}$ based design example. The matrix $\boldsymbol{G}_{2 \times 4}$ is given in (11), while the matrix $\boldsymbol{G}_{4 \times 8}$ obtained from a systematic generator matrix of a ReedSolomon code is presented in [5], [6].

The $\boldsymbol{G}_{2 \times 4}$-based system is represented by ( $R=0.5, M=2, k_{1}=$ $\left.1, k_{2}=1, \boldsymbol{G}_{2 \times 4}, R_{\text {info }}=0.5,3 \leq D_{(2 \times 4) N N C N M} \leq 3\right)$, and the 
$\boldsymbol{G}_{4 \times 8}$-based system is characterised by ( $R=0.5, M=2, k_{1}=$ $\left.2, k_{2}=2, \boldsymbol{G}_{4 \times 8}, R_{\text {info }}=0.5,4 \leq D_{(4 \times 8) N N C N M} \leq 5\right)$. The two systems are comparable, since they both have the same $R, M$ and $R_{\text {info }}$ values. However, the more complex transfer matrix $\boldsymbol{G}_{4 \times 8}$ has a higher diversity order of $4 \leq D_{(4 \times 8) N N C N M} \leq 5$ (as opposed to $\left.3 \leq D_{(2 \times 4) N N C N M} \leq 3\right)$. This also means that the $\boldsymbol{G}_{4 \times 8}$-based system is expected to have a superior FER performance in comparison to the $\boldsymbol{G}_{2 \times 4}$-based system.

\section{Simulation Results AND Discussions}

Let us now consider the simulation results presented in Fig. 5, where the number $N=1$ sub-frame used in [5], [6] is employed. It can be seen from Fig. 5 that the difference in the diversity order of the $\boldsymbol{G}_{2 \times 4}$ and $\boldsymbol{G}_{4 \times 8}$ based systems, as specified in Section V-B, is reflected by the different slope of the performance curves.

Fig. 5 substantiates our analysis provided in Section IV-B, where the performance estimate found with the aid of the PRBM was always superior, but optimistic in comparison to that obtained by the actual simulations. More explicitly, it is shown in Fig. 5 that their deviation was found to be from $0.3 \mathrm{~dB}$ to $0.5 \mathrm{~dB}$ at an FER of $10^{-4}$.

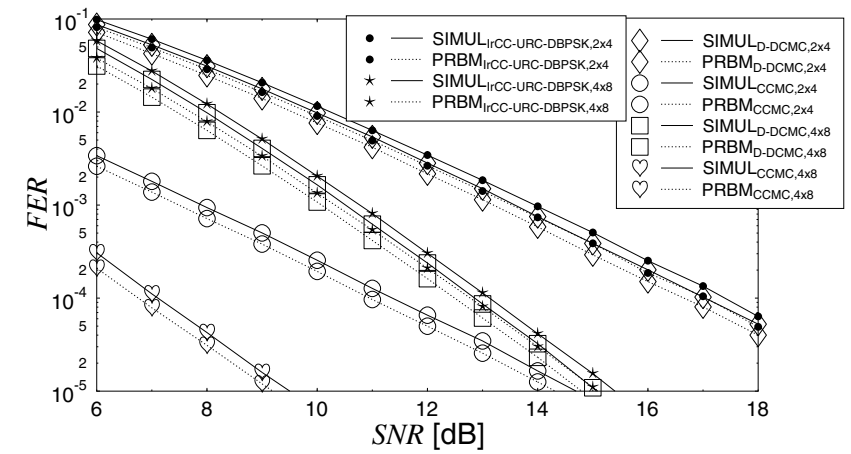

Fig. 5. FER performance comparison between $\boldsymbol{G}_{2 \times 4}$ and $\boldsymbol{G}_{4 \times 8}$ based systems employing the IrCC-URC-MSDD-aided-DBPSK scheme and idealised/perfect CCMC and D-DCMC channel coding schemes when having the number $N=1$ of sub-frames.

Fig. 6 characterises the FER performance of the systems obtained by the actual simulations, when the number of sub-frames increases from $N=1$ to $N=100$. The combination of sub-frame based transmissions and network coding is capable of providing the $11 \mathrm{~dB}$ (or $8 \mathrm{~dB}$ ) performance improvement, when comparing the $\boldsymbol{G}_{2 \times 4}$ (or $\boldsymbol{G}_{4 \times 8}$ ) based systems employing either $N=1$ or $N=100$ subframes at an $F E R$ of $10^{-3}$. Another important result gleaned from Fig. 6 is that if either the network coding used or the number of subframes $N$ is given, we can optimise the other of the two parameters, so that the system can achieves its best possible performance.

As seen in Fig. 5 and Fig. 6, the performance of the $\boldsymbol{G}_{4 \times 8}$ and $\boldsymbol{G}_{2 \times 4}$-based systems using our IrCC-URC-MSDD-aided-DBPSK scheme was within $0.3 \mathrm{~dB}$ to $0.5 \mathrm{~dB}$ from that of the corresponding systems relying on the assumption of using an idealised/perfect DDCMC channel coding scheme at an FER of $10^{-4}$.

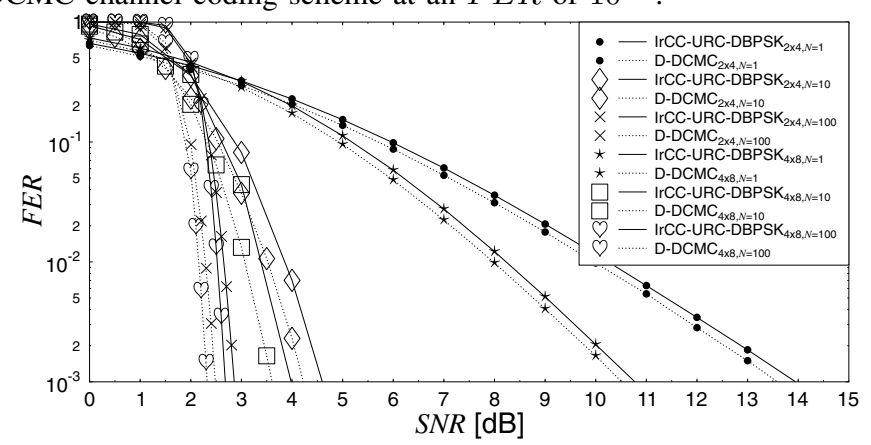

Fig. 6. FER performance comparison between $\boldsymbol{G}_{2 \times 4}$ and $\boldsymbol{G}_{4 \times 8}$ based systems employing the IrCC-URC-MSDD-aided-DBPSK scheme and idealised/perfect D-DCMC channel coding schemes supposedly employing the different number of sub-frames $N=1,10,100$.

\section{CONCLUSIONS}

In this contribution, we investigated new Near-capacity Noncoherent Cooperative Network-coding aided Multi-user systems using our IrCC-URC-MSDD-aided-DMPSK scheme, which was designed with the aid of EXIT charts. The achievable performance was benchmarked against the corresponding systems employing the idealised/perfect channel coding schemes assumed to be operating exactly at the D-DCMC capacities.

\section{REFERENCES}

[1] R. Ahlswede, N. Cai, S.-Y. Li, and R. Yeung, "Network information flow," Information Theory, IEEE Transactions on, vol. 46, pp. 1204 1216, jul 2000.

[2] A. Asterjadhi, E. Fasolo, M. Rossi, J. Widmer, and M. Zorzi, "Toward network coding-based protocols for data broadcasting in wireless ad hoc networks," IEEE Transactions on Wireless Communications, vol. 9 , pp. $662-673$, Feb. 2010.

[3] M. Xiao and M. Skoglund, "M-user cooperative wireless communications based on nonbinary network codes," in IEEE Information Theory Workshop on Networking and Information Theory, 2009. (ITW 2009), pp. $316-320$, June 2009.

[4] M. Xiao and M. Skoglund, "Multiple-user cooperative communications based on linear network coding," IEEE Transactions on Communications, Dec. 2010

[5] J. L. Rebelatto, B. F. Uchôa-Filho, Y. Li, and B. Vucetic, "Generalized distributed network coding based on nonbinary linear block codes for multi-user cooperative communications," in 2010 IEEE International Symposium on Information Theory (ISIT 2010), pp. 943 -947, June 2010.

[6] J. L. Rebelatto, B. F. Uchôa-Filho, Y. Li, and B. Vucetic, "Multiuser cooperative diversity through network coding based on classical coding theory," Submitted to IEEE Transactions on Information Theory, [Online]. Available: http://arxiv.org/abs/1004.2757.

[7] M. Tuchler, "Design of serially concatenated systems depending on the block length," IEEE Transactions on Communications, vol. 52, pp. 209218, Feb. 2004.

[8] S. ten Brink, "Convergence behavior of iteratively decoded parallel concatenated codes," IEEE Transactions on Communications, vol. 49, pp. 1727-1737, Oct. 2001

[9] P. Ho and D. Fung, "Error performance of multiple-symbol differential detection of PSK signals transmitted over correlated Rayleigh fading channels," IEEE Transactions on Communications, vol. 40, pp. 1566 -1569 , Oct. 1992.

[10] V. Pauli, L. Lampe, and R. Schober, “'Turbo DPSK” using soft multiplesymbol differential sphere decoding," IEEE Transactions on Information Theory, vol. 52, pp. 1385 - 1398, Apr. 2006.

[11] S. X. Ng, J. Wang, M. Tao, L.-L. Yang, and L. Hanzo, "Iteratively decoded variable length space-time coded modulation: Code construction and convergence analysis," IEEE Transactions on Wireless Communications, vol. 6, pp. 1953-1963, May 2007.

[12] D. Tse and P. Viswanath, Fundamentals of Wireless Communications. Englewood Cliffs, NJ, USA: Cambridge: Cambridge University Press, 2005.

[13] C. F. Golub, Gene H.; Van Loan, Matrix Computations. Johns Hopkins Studies in Mathematical Sciences: Johns Hopkins University Press, 1996.

[14] L. Kong, S. X. Ng, R. Tee, R. Maunder, and L. Hanzo, "Reducedcomplexity near-capacity downlink iteratively decoded generalized multi-layer space-time coding using irregular convolutional codes," IEEE Transactions on Wireless Communications, vol. 9, pp. $684-695$, Feb. 2010.

[15] L. Hanzo, O.Alamri, M. E.Hajjar and N.Wu, Near-Capacity MutiFunctional MIMO Systems. New York, USA : John Wiley and Sons, 2009.

[16] A. Ashikhmin, G. Kramer, and S. ten Brink, "Extrinsic information transfer functions: model and erasure channel properties," IEEE Transaction on Information Theory, vol. 50, pp. 2657-2673, Nov. 2004.

[17] H. Ochiai, P. Mitran, and V. Tarokh, "Design and analysis of collaborative diversity protocols for wireless sensor networks," in Proc. VTC2004 Fall Vehicular Technology Conference 2004 IEEE 60th, vol. 7, pp. 46454649, Sept. 26-29, 2004. 